\title{
Einige Termini der lappischen Mythologie im sprachgeographischen Licht
}

\author{
Von Olavi Korhonen
}

Die ältesten schriftlichen Quellen der lappischen Religionsforschung sind auch von Bedeutung für Untersuchungen der sprachlichen Verhältnisse unter den Lappen in alter Zeit. Auch wenn der Sprachforscher nur einzelne lappische Wörter und Ausdrücke in dem ältesten schriftlichen Material findet, haben diese dennoch einen grossen Wert, weil sie üblicherweise in einem Zusammenhang stehen, der deutlich die Bedeutung der Wörter zeigt. Mit Kenntnis des Verfassers und seines Wirkungsortes können die Termini oft einem bestimmten Dialektgebiet zugeführt werden. Das letztgenannte Kriterium kann jedoch manchmal irreführend sein, weil der Textverfasser, ohne seine Quelle anzugeben, Angaben von ganz anderen Gegenden wiedergeben konnte. Durch die Lautgestalt oder die Formen, in denen die lappischen Wörter wiedergegeben werden, kann so ein Fall manchmal blossgelegt werden. Es sagt sich von selbst, dass eine Kontrolle der Provenienz der religionsgeschichtlich wichtigen Termini eine dringende quellenkritische Frage ist.

Was man zuerst tun sollte, ist, die lappischen Wörter und Ausdrücke, die in einer Quellenschrift vorhanden sind, miteinander zu vergleichen, um zu sehen, ob sprachliche Einheitlichkeit vorliegt. Für die Entscheidung, ob die Wörter von einem und demselben Sprachgebiet stammen, oder ob sie von verschiedenen Gegenden gesammelt sind, kann die Lautgestalt der Wörter von Bedeutung sein. Die instabile Schreibung in den Texten bereitet jedoch oftmals Schwierigkeiten. Kasusformen (z. B. südliches $-n$ für Genitiv Singular in zusammengesetzten Substantiven) und Nomen- oder Verbableitungen mit mehr oder weniger charakteristischer geographischer Verteilung können auch vorkommen. Gewöhnlicherweise fehlen aber Kriterien dieser Art für eine geographische Ortung der lappischen Elemente eines Textes. Es gibt jedoch noch eine andere Methode, die sprachliche Provenienz zu bestimmen.

Die Methode, an die ich denke, ist gelegentlich sowohl von Sprachwissenschaftlern als auch Religionshistorikern angewendet worden und sie geht von den Möglichkeiten aus, in den jetzt existierenden lappischen Dialekten Parallelen zu den auftretenden lappischen Termini zu finden und 
ihre jetzige Verbreitung zu kartieren. Der Wortschatz des Lappischen hat sich im Laufe der Jahrhunderte stark verändert, und Termini mit Anknüpfung an die lappische Religion sind oft in Vergessenheit geraten, aber die Methode kann für noch existierende Termini trotzdem fruchtbringend sein. Die sprachlichen Parallelen, die schlimmstenfalls sich vielleicht nur noch in dem lappischen Ortsnamenmaterial finden, machen jedoch ein Komplement zu den kurzgefassten alten Texten aus.

Wenn K. B. Wiklund - in seiner bekannten Arbeit von 1916 über die Vorstellungen der Lappen vom ,Saivovolk“ - die variierenden Bezeichnungen, die die Unterirdischen in verschiedenen Gegenden haben, durchgeht, dann scheint oft die Betrachtungsweise des Sprachwissenschaftlers durch. Man müsse, meinte er, als erstes, die geographische Provenienz der Quellen erorten, welches manchmal schwer genug sein kann und im allgemeinen allzusehr vernachlässigt worden ist" ", und er betont, wie ,, notwendig und unumgänglich eine eingehende philologische Analyse bei der Behandlung eines religionshistorischen Problems sei, auch wenn es sich um eine Religion eines Naturvolkes handele" (Wiklund 1916, 46).

Wiklund stellt generell fest, dass die Saivo-Termini und -Vorstellungen der norwegischen Quellen zu, ,den südlichsten lappischen Gegenden sowohl in Schweden als auch in Norwegen gehören " (vgl. Bäckman 1975) und gar keine terminologischen Verankerungen in der Finnmark haben (Wiklund 1916, 58). Zu Fellman und Castrén sei Saivo als Name für ein geistliches Wesen auf literarischem Wege gekommen (Wiklund 1916, 62), und das bei Jessen vorkommende Saiwo-olmak mit nordlappischer Pluralendung (-k) sei ausserdem auf fehlerhafte Generalisierung zurückzuführen, wie Wiklund es nennt.

Wir wissen aus vielen Beispielen, dass Friis in sein Wörterbuch (Friis 1887) Wörter aus entlegenen Dialekten (auch aus dem Lexicon Lapponicum von Lindahl \& Öhrling, 1780, das vorwiegend Ume- und Pite-lappische Gebiete in Schweden behandelt) aufnahm, ohne den Ursprung anzugeben. Das gilt offenbar auch für das Wort Saivvo ,, der Platz unter der Erde, wo die Lappen glaubten, dass sich die glücklich Verstorbenen mit neuen Leibern aufhalten und das selbe Gewerbe wie hier auf Erden treiben würden"' (Friis 1887, 601).

Manchmal ist es möglich, die geographische Herkunft der Wörter mit Hilfe der Lautgestalt herauszufinden. In anderen Fällen kann die Bedeutung in verschiedenen Gegenden hilfreich sein. Was das Wort (saN) sái'va (mit Varianten, aber gewöhnlicherweise mit kurzem - $a$ in der zweiten Silbe) betrifft, kann man feststellen, dass die nichtmythologische Bedeutung 'Süsswasser' in ost- und zentrallappischem Gebiet, d. h. auf der Kolahalbinsel und in den nördlichsten Teilen von Finnland, Norwegen und Schwe- 
den, vorherrscht. Von der westlichen Finnmark ab und in Richtung Südwesten tritt die Bedeutung 'heiliger See' immer mehr hervor, und von den mittleren Lappmarken ist sie der einzige Rest einer früheren nichtmythologischen Bezeichnung für kleinere Seen im allgemeinen. Das Wort Saivvo bei Friis ist deswegen sowohl wegen der Lautgestalt (mit $-o$ ) als auch wegen der Bedeutung irreführend. Trotzdem hat es einen Stammkonsonanten im Starkstadium (-ivv-) erhalten, als wenn es in den Mundarten der Finnmark belegt wäre. Ein unkritischer Benutzer des Wörterbuches kann leicht dazu verleitet werden, auf Grund des Wortartikels eine falsche Theorie aufzubauen.

Wiklunds Einschätzung der Verbreitung der Termini fusste auf eigenen Aufzeichnungen oder Aufzeichnungen anderer, aber er hatte nicht zu so guten Lexika oder Wortsammlungen Zugang wie wir jetzt. Zu der Reihe grösserer lappischer Wörterbücher gesellt sich jetzt auch ein südlappisches Wörterbuch von Gustav Hasselbrink, vom Institut für Mundarten und Volkskunde in Uppsala herausgegeben. Die Möglichkeiten, innerhalb des südlichen lappischen Wortschatzes dialektgeographische Studien zu betreiben, sind deswegen erheblich verbessert worden. Noch würden jedoch mehr leichter zugängliche Wortsammlungen über enare-, torne- und umelappische Gebiete benötigt.

Wenn wir uns noch einen Augenblick bei Wiklunds Artikel über Saivo von 1916 aufhalten, bemerken wir die Einstellung, die er als Sprachwissenschaftler zu seinem Thema hat. Angehend die Vorstellungen der Lappen, die an die ,Unterirdischen“ geknüpft sind, beachtet er die Bezeichnungen für die Erdgeister und die geographische Verbreitung der Termini. So weit die Unterirdischen Namen z. B. nordischer Herkunft haben, kann man deutlich getrennte Distributionen der betreffenden Termini finden. Ein Beispiel ist das ' $g o(d) v e t t e r$ ' der Norweger, was vorwiegend in einem östlichen Teil des Zentrallappischen in der Form (saN) gufittar (vgl Wiklund 1916, 49; Itkonen 1946, $92 \mathrm{ff}$.) zu finden ist, während die lappische Form (saN) $u l^{\prime} d a$ (vom schwedischen 'huldra') vorwiegend in den westlichen Teilen des Nordlappischen vorkommt. Weiter östlich finden sich Belege für den ersteren Terminus auf jeden Fall von Karasjok (Wiklund 1916, 51) und Utsjoki, aber in dem späteren Falle mit einer entgleisten Bedeutung (d.h. einer Bedeutung, die sich stark von der ursprünglichen entfernt hat) ,unhöfliche, schweigsame Person“" (Ravila 1934, 101). Im Westen erreicht das Wort hinwieder u. a. die Ofoten (Qvigstad 1893, 182). T. I. Itkonens Bemerkung, dass $u l^{\prime} d a$ zu der genuinen Terminologie in Enontekiö gehört, während gufittar nur in gewissen festen Redewendungen gebraucht wird, veranschaulicht, wo das Grenzgebiet zwischen den Wörtern verläuft (Itkonen 1946, 96). Offenbar späte Varianten, die mit $h$ - (hul'da, holdo, 
holldo) anfangen, erwähnt Qvigstad von Gebieten in Norwegen auf der Höhe von lule- und südlappischen Dialektgebieten (Qvigstad 1893, 337), und einen naheliegenden Beleg gibt es auch von Arjeplog (vgl. Wiklund 1916, 53). Wenn folglich ein Einfluss vom Norwegischen in einer östlichen Hälfte des zentrallappischen Gebietes und Teile des Ostlappischen mit der langen Küstenstrecke im Western vorherrschend $\mathrm{zu}$ sein scheint (gufittar), so ist offenbar schwedischer Einfluss, ausser in Torne-Lappmark in Schweden, auch in den entsprechenden Gebieten in der westlichen Finnmark bemerkbar $\left(u l^{\prime} d a\right)$.

Alte politische Verhältnisse können für diese Terminusverbreitungen die Erklärung sein. Einen anderen schwedischen Einfluss finden wir in den Termini vittarah, vitara (=viihtarah) aus Arjeplog (Wiklund 1916, 53; Kolmodin 1914, 30; Dahlstedt 1976, 17, 46). Dafür habe ich auch durch eigene Aufnahmen mit Lappen in den betreffenden Gebieten Belege.

Weitere Termini könnten erwähnt werden, aber hier ist nur die Absicht, gewisse Tendenzen in ihren Verbreitungen vorzuweisen. Einen ganz anderen dialektgeographischen Charakter hat ein wohlbekanntes und ursprüngliches lappisches Wort für die Unterirdischen. Ich denke an ganii, Pl. gadnihah, um nun die Aussprachevariante, die in den zentralen Teilen des Lulelappischen vorherrschend ist, zu erwähnen (Grundström 1946-54, 165). Wenn man in den mittleren Lappmarken in Schweden von älteren lappischsprechenden Personen noch Vittersagen $\mathrm{zu}$ hören bekommen kann, wird gerade dieses Wort gebraucht. Wo es die früher erwähnten entliehenen Bezeichnungen gibt, ist dieser ältere Terminus ganz offenbar verdrängt worden. Zwischen Wörtern, die dieselbe Erscheinung bezeichnen, besteht oft ein Kampf um die Stellung innerhalb des Wortschatzes eines Idioms (d.h. eines Dialekts). Der Ausgang eines solchen Zweikampfes kann wichtige Informationen nichtlinguistischer Art beinhalten, wie z. B. die Art der ethnischen Kontakte in einer Gegend, die Richtung und die Stärke von Einflüssen u. s.w. Man kann sich hier fragen, warum einige nordische Termini so grosse Verbreitung im Norden erhalten haben, während ein lappischer Terminus von ursprünglichem Charakter in grossen Teilen der Schwedischen Lappmarken standgehalten hat. Die wortgeographischen Verhältnisse verlocken uns dazu, die dahinterliegenden Ursachen zu suchen; diese sind jedoch selten leicht eindeutig aufzudecken.

Bei den Vorstellungen von den Unterirdischen können wir auf jeden Fall sehen, dass die ursprünglichen Termini in Vergessenheit geraten oder in ihrer Bedeutung stark verändert worden sind, wenn es fremden Termini gelungen ist einzudringen.

Die Entsprechung zu dem ganii, Pl. gadnihah, des Lulelappischen findet sich in dem Wörterbuch von Leem (1768-1781) in der Form ganish, Pl. 
ganishjak,,et Bierge-Trold, spectrum, qvod vulgo crebedatur montes incolere" (vgl. Nielsen \& Nesheim 1962, 29). Wie man es von einem alten Terminus für eine volkstümliche Vorstellung erwarten kann, tritt diese Bezeichnung vereinzelt über ein grosses Gebiet auf. Ich sehe jedoch einen Zusammenhang zwischen der Tatsache, dass der Terminus im Norden in späterer Zeit so selten vorkommt, und dem Umstand, dass entlehnte Wörter für die Unterirdischen dort so grosse Verbreitung bekommen haben. Die Wörter gäniš, Pl. gäničak (auch -æ-) ,,ecko, Ekko; daemon montanus, Trold, som bor i Fjeldvægge" bei Friis (Friis 1887, 276) und găneš, Pl. gănečak,,daemon montanus" laut einer Aufzeichung in Kvänangen von Qvigstad (siehe Wiklund 1916, 50) haben den Stempel der Ursprünglichkeit, aber piru kēneš, ein Kraftausdruck für Mensch oder Tier bei Gebirgslappen in Enare (Itkonen 1946, 70), Kinnaš-Elle (Kvänangen; Lagercrantz 1939, 298) ,,eine kleine Frau“" und geniš, als Bezeichnung für eine schwerhörige Person in Nesseby, haben sich schon von ihrem Platz in Mythologie und Folklore entfernt. Dasselbe gilt in gewisser Hinsicht für kanis, Pl. katnihah, in Jukkasjärvi, was die Geister der Toten, die man auf andere Menschen setzen oder ihnen senden und sie dadurch verrückt machen kann, bezeichnet (Wiklund 1916, 51). Hier hat der Terminus ganz seine Anknüpfung an hauptsächlich positiv erwähnte ,,Unterwohner“, Unterirdische, oder wie wir sie nennen wollen, verloren und ist statt dessen eine Bezeichnung für dämonische Geister oder Kräfte, die übel gesinnte Personen ihren Gegnern senden können, geworden. Der Glaube an 'sändingar' (saN, Pl. bijahat) einer bösen Kraft, von einem Geist oder ähnlichem, den man durch Zauberei sendet und der einen Menschen krank oder geisteskrank macht, von dem man bei der Feldarbeit im Norden ständig volkstümliche Erzählungen erhält, macht ein kräftiges Attraktionszentrum aus. Hier sehen wir ein deutliches Ergebnis der Anziehungskraft, die diese Vorstellung ausgeübt hat.

Südlich von den eben genannten Gebieten, das heisst in Lule-Lappmark im weitesten Sinne, begegnen wir dann ganii, Pl. gadnihah 'vittra' (Grundström 1946-1954, 165; oder Formen, die vom Umlaut beeinflusst sind wie genii, Pl. gednihah 'vittra'; Collinder 1938, 60 Waltlappisch in Gällivare), ginii (Grundström \& Väisänen 1958, 58) kinê, Pl. kitniha 'unterirdisches menschenhaftes Wesen' (Arjeplog; Halász 1896, 28), Nom. Sg., kinegi 'Heinzelmännchen' (Malå; Wiklund 1916, 53) u. s. w. alle mit Bedeutungen, die wir mit lappischem Glauben an die Unterirdischen in Verbindung bringen können. Es gibt viele Erzählungen (Dahlstedt 1976, $15 \mathrm{ff}$.), aber deren Inhalt gehören nicht zu dem Thema dieser Arbeit, deswegen werden sie hier übergangen (siehe hierzu Wiklund 1916, 52). Bei Interviews mit älteren Informanten oder Informanten mittleren Alters kann 
man noch heutzutage selbsterlebte Ereignisse zu hören bekommen, laut welchen die Unterirdischen sich vorwiegend als Warnzeichen vor den ermüdeten Rentreibern oder Waldleuten im allgemeinen vor nahendem Unwetter zeigten. Die Sagen mit ihren bekannten Bildern sind natürlich noch häufiger.

Für den Sprachwissenschaftler ist es interessant, die in grossen Gebieten vorkommenden gemeinsamen Bilder, die keine Entsprechungen in einer besonders grossen Einheitlichkeit in der Terminologi haben, zu vergleichen.

Bei einer weitergehenden Reise südwärts über die Dialektkarte zeigt sich dieses nochmals deutlich. Ausser dem Malålappischen kitnegi in der Bedeutung 'Heinzelmännchen' finden wir das Wort mancherorts im Südlappischen in Jämtland schwach belegt (u. a. gidnj'ese, -sje, giqnj'esje 'eine Art Geister; Menschen die überall lauernd und spähend umherfahren; Hasselbrink 1983, 587; vgl. Wiklund 1916, 56). Von einer stärkeren Stellung des Wortes in älterer Zeit zeugen solche Ortsnamen wie Gitnivaratje (eigentlich 'Der kleine Vitterberg', Lillberget in der Nähe vom westlichen Kikkejaur) in Arvidsjaur und Kitnihasse (Viterliden) in Malå. Wenn ein Dialektwort aus dem Wortschatz der Bevölkerung verschwindet, kann es für unüberschaubare Zeit in den Ortsnamen bestehen bleiben. So ist es in den eben genannten Teilen des Nordlappischen der Fall gewesen, wo Namen wie Kenešpahta (Utsjoki), Kenisjärvi (Kittilä) und Kinislampi (Rovaniemi) von einem früheren Gebrauch dieses Wortes für die Unterirdischen zeugen (Wiklund 1916, 48).

Hier können wir auf die Relation zwischen Termini, die in älteren schriftlichen Dokumenten bewahrt sind, und Termini, die in der lebendigen Sprache zu finden sind, eingehen. Im Nærömanuskript von 1723 erwähnt Johan Randulf eine Waldgöttin mit dem Namen Gidne, die alle Kennzeichen der Waldfrau trägt. Ihre Vorderseite war schön, aber hinten trug sie einen Schwanz und sie war trügerisch gegen die Männer im Walde (Kildeskrifter 1903, 46). Die Figur ist offenbar von nordischer Herkunft, aber der Terminus ist lappisch. Der Einschub - $d$ - (gidne statt gine) gehört mit süd- und umelappischem Lautbild zusammen. Der Verfasser des Nærömanuskripts kann deswegen den Terminus zum Biespiel nicht vom Norden geliehen haben. Der Ort für die Aufzeichnung (Bäckman 1975, 30f., 36) liegt auch auf der Höhe vom nördlichen Südlappischen in Schweden und alles deutet darauf hin, dass dieses ein genuiner Beleg von der lappischen Bevölkerung des Ortes ist.

Aber was ist dann der Grund dafür, dass der in Lule-Lappmark wohlbekannte Begriff ganii (mit Varianten) im Süden so schwach belegt ist? Der Grund ist offenbar hier derselbe wie im Norden: Konkurrenz von anderen 
eindringenden Termini. Das im Süden wohlbekannte saivo saiva, was übrigens ein häufiger Terminus in Randulfs Text ist, ist im Laufe der Zeit ganz dominierend geworden. Das ist der sprachliche Faktor, der bewirkt, dass unsere Terminuskarte so aussieht, wie sie es tut.

Ein wortgeographisches Studium kann folglich allerhand darüber aussagen, wie sich das Traditionsgut einer Volksgruppe entwickelt hat. Wortverteilungen und der Ursprung einzeler Wörter und Termini - in den Fällen, wo sie entlehnt sind und nicht den Charakter von ererbten Grundwörtern haben - haben viel über Kontakte zwischen den Völkern zu berichten. Der Bruch zwischen Ursprünglichem und Neuem, für den ich hier Beispiele habe geben wollen, ist interessant zu studieren. In meinem obigen übersichtlichen Durchgang habe ich die Verbreitung der Termini nicht mit den vorhandenen Dialektgrenzen verglichen. Mehrere auffällige Übereinstimmungen können jedoch festgestellt werden und an einer Traditionsgrenze werde ich mich noch weiter aufhalten, nämlich der, die den Terminus ganii (mit Varianten), der noch heute in lappischer Folklore eine starke Stellung hat, von saiva saivo trennt. Das erstere Wort findet sich in den Gemeinden von Arjeplog und Jokkmokk und in den Gebieten nördlich davon, das letztere kennen wir von Tärna, Sorsele und Stensele (d.h. ungefähr Västerbotten) und südwärts (vgl. Bäckman 1975, 132). Hier verläuft auch eine wichtige Sprachgrenze, nämlich die zwischen südlicherem beziehungsweise nördlicherem Lappisch im weitesten Sinn auf schwedischem Gebiet.

Ich werde noch ein weiteres Beispiel dafür geben, wie diese Grenzzone hervortritt. Um einen Kontrast zu dem so oft studierten nordischen Einfluss zu erhalten, wähle ich einen Terminus von östlicher Herkunft. Östliche Entlehnungen sind in nördlichen lappischen Dialekten häufig, aber dagegen mit einer ausschliesslichen Verbreitung im Süden selten. Das Dokument, aus welchem der betreffende Terminus geholt worden ist, ist Epitomes historiae missionis Lapponicae pars prima von Hans Skanke (Skanke 1945, 179ff.), das irgendwann nach dem Tod von Thomas von Westen 1727 verfasst worden ist (Qvigstad 1943, 37f.). Nach einer Beschreibung der drei Arten von 'Creature' die der Zauberer in seinem 'Nåjden-Dienst' hat, nämlich Vogel, Fisch und Ren-Stier, findet Skanke es geeignet, die Namen der Vögel zu erwähnen, die ihrem Besitzer folgen und ihm in Jagd und Renzucht beistehen, die mit Botschaften kommen oder fahren und so weiter. Die Liste enthält siebzehn Namen, von welchen Qvigstad acht identifiziert hat. Ein Vogelname ist Jap (Skanke 1945 191), der als das Südlappische jipp, jihpa 'the great horned owl' gedeutet worden ist (Qvigstad 1943, 45). In dem neuen Südlappischen Wörterbuch finden sich Belege für jüppe von Vefsn, Vilhelmina, Frostviken, Snåsa, 


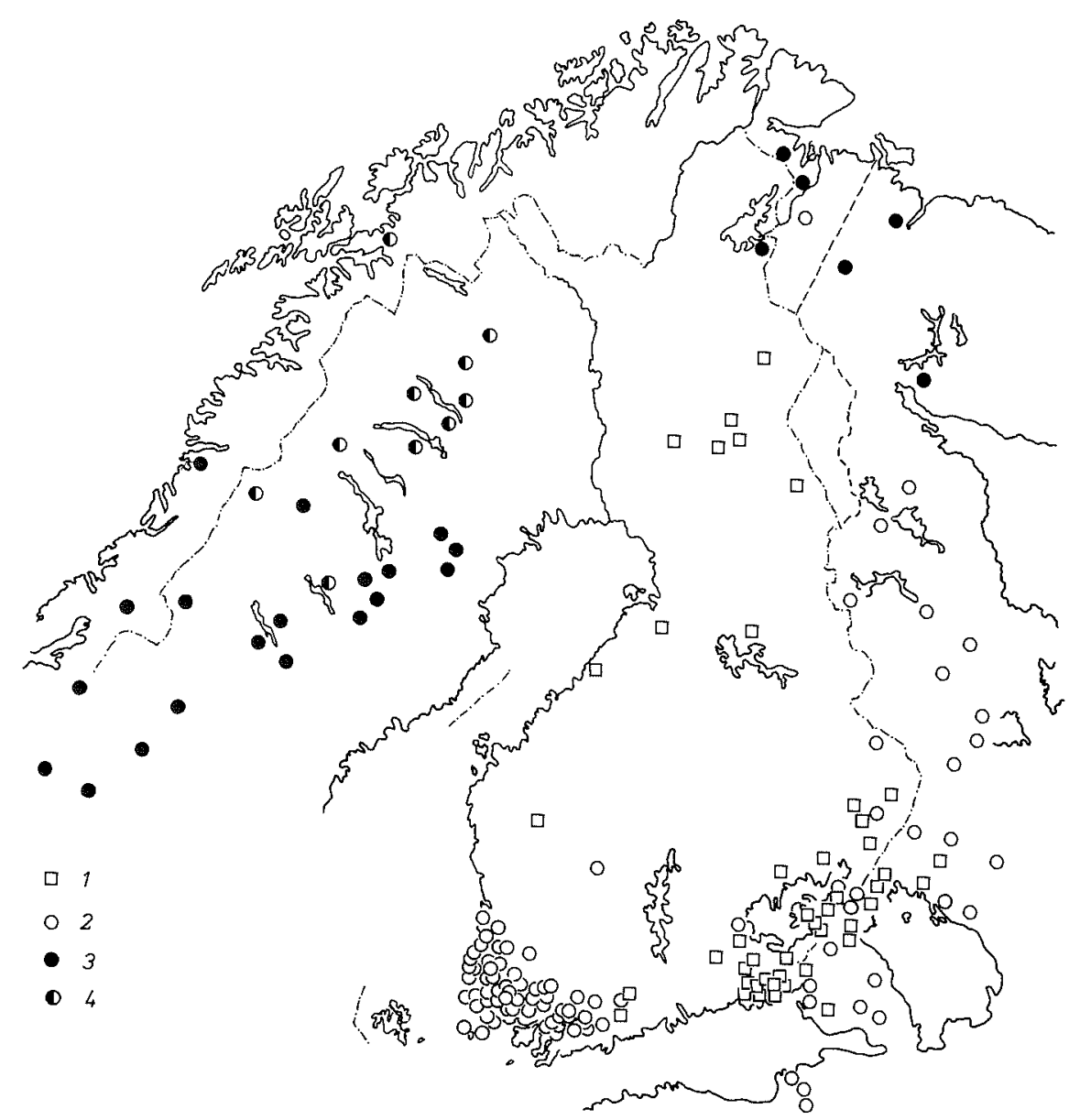

Fig. I. 1. Finnische und karelische Ortsnamen, die auch den Benennungen des Berguhus zurückgehen (Hüpiä-, Hüpiö- etc.). 2. Finnische und karelische Belege für die Benennungen des Berguhus (hyypiö, -piä, -ppiö, -ppiä resp. hyypie, hyybie etc.). 3. Das lappische Wort für den Berguhu (im westlappsichen jippege, jippelii, jippil, jippa etc; siehe oben genannte Wörterbücher bezüglich den östlichen Belege). 4. Das westlappische Wort in einer anderen Bedeutung als 'Berguhu' (siehe Text).

Meraker, Offerdal, Oviken in der Bedeutung 'Bergeule, (Gebirgs-)uhu', oft auch 'eine graubraune Eule, Horneule'. Die Form jïppege findet sich in Röros und Tännäs (Härjedalen), hier als 'Baum-, Waldkauz', ausserdem an dem jeweiligen Ort in der Bedeutung 'dummer Mensch (der albern lacht); (der) Heide'; 'ungewöhnliche Erscheinung, Gespenst' (Hasselbrink 1983, 792). Ausser diesen Wörterbuch-Angaben habe ich aus Vilhelmina in der 
Handschrift von J. A. Nensén (UUB R 649, 1) den Beleg jipp (Gen Sg. 1) jip̄eken 'Uhu'.

Interessant ist die Frage der weiteren Verbreitung des Wortes zum Beispiel im Umelappischen, welches das nördlichste Gebiet des Südlappischen ist, vom Umeälv hoch bis zu den Gemeinden Malå, Arvidsjaur, Sorsele und dem nördlichen Tärna. Aus zugänglichen Wörterbüchern sehen wir, dass das Wort im Gebiet bekannt ist: jipp 'Bergeule (Lindahl \& Öhrling $1780,93,100,237) \sim$ jippa (Lindahl \& Öhrling 1780, 696). Synonyme sind laut Lindahl \& Öhrling lidno und maiwe, für welche ich im Umelappischen Belege habe. Von Malå stammt Schlachters jihpagii, jihpaga, 'Uhu' (Schlachter 1958, 73).

Von dem Vorkommen des Terminus in der Literatur und von meinen Exzerpten kann eine gleichmässige Verteilung im übrigen Umelappischen bestätigt werden. Belege, die an bestimmte Gemeinden gebunden sind, sind unter anderen: (Tärna) jeppok '(Kinder die) nichts gesehen haben, gehört oder gelernt haben (und die für den Mangel an guten Sitten getadelt werden)' (Pettersson 1979, 159), (Stensele, Ullisjaur) ǰ̌cpaka 'Zauberfisch' (DAUM 3844, A. Calleberg 1925-26), (Sorsele, Ranbyn) jipp 'Uhu' (UUB R 649, 359, J. A. Nensén) und jixp(a)ka, jix̄'paka, jixpak 'Uhu' (ULMA 3032: 2, A. Calleberg 1930), (Arvidsjaur, Lomträsk) jih̀pâGij 'Berguhu' (ULMA 16359, B. Collinder 1943), (Arvidsjaur, Fjällbonäs) jippege 'etwas eigenartig(-e) Person)' (DAUM 3793, I. Ruong), (Malå, Släppträsk) jix̄pagə 'Uhu' (DAUM 3622, K. B. Wiklund 1900). Ohne nähere Ortung ist Nenséns Angabe jipp 'Uhu' von Lappmarken von Åsele und Lycksele (UUB R 649, J. A. Nensén, 215, 414). Von der lappischen Siedlung Stenbacken in Arvidsjaur habe ich jippege 'Berguhu' aufgezeichnet (Jonas Jonsson 800531) und in Arjeplog, Stenbacken, den Beleg jippa 'Gewitterwolke' (DAUM 3398, 800) mit Sara Ruong auf Band aufgenommen.

Näher an der Gebirgskette neigt das Wort dazu, eine abweichende Bedeutung zu erhalten (entartetes Kind in Tärna, Zauberfisch in Stensele und Gewitterwolke in Arjeplog), aber dieses ist für die einzelnen Belege, die nördlich des Umelappischen zu finden sind, noch deutlicher. Aus Arjeplog gibt es nur ein Beispiel, aber im nördlichen und zentralen Jokkmokk bedeutet jippelii, (jippelij jippil, jippilih-) 'Kosekind (kleines Kind)', im nördlichen Gällivare ist jippelii 'das versteckte Kind von Eva, das von Gott verfluchte u.s.w.' und im südlichen und nördlichen Teil der Gemeinde ist jippa 'schwarze Gewitterwolke' (Grundström 1946-54, 165). Obwohl ich Informanten im Gebiet das Wort oft aktualisiert habe, habe ich nur von Bieggaluokta, Porjus, jippelahah (Pl.) in der Bedeutung 'kleine Kinder (die zum Beispiel auf dem Hof spielen und toben'; 810816). Man kann verstehen, dass Wiklund (Wiklund 1916, 51) sich gegenüber einer 
Angabe bei Fellman (Fellman 1903, 109) fragend stellt, laut welcher der Terminus Jppelak (Offenbar P1.) in Jukkasjärvi dasselbe die Kanesak sein, also die Unterirdischen bezeichnen sollte. Wiklund hatte im Norden keine Parallele zu dem Terminus.

Jetzt hat jedoch Qvigstad in Ibestad in Norwegen, auf der Höhe des tornelappischen Gebiets in Schweden, wo Jukkasjärvi liegt, ippel, Pl., ippelah 'etwas Übernatürliches (gehört)' aufgezeichnet, bzw. ippelaš, P1., (G)ìppelažžak (OUB 1465e, J. Qvigstad). Wie wir sehen, hat sich die Bedeutung bei all den selten vorkommenden Belegen nördlich von der lappischen Sprach- und Traditionsgrenze, die ich mehrmals erwähnt habe, stark von der ursprünglichen Bedeutung entfernt. Wir können von regelrechten Bedeutungsentgleisungen sprechen, was für Wörter ausserhalb ihrer Kerngebiete charakteristisch ist.

Welches ist dann die Etymologie des Wortes? Wie ich schon erwähnt habe, handelt es sich um ein Wort östlichen Ursprungs. Das Wort kann laut dem Finnischen Etymologischen Wörterbuch (SKES) einem finnischen Wortstamm zugeführt werden. Es hat auch einige ostlappische Dialekte erreicht und findet sich im Enarelappischen (opp' $u \bar{u}$ ), ausserdem ist es in Kuolajärvi (dem jetztigen Salla; hubbug) aufgezeichnet worden.

Wir finden, dass der Vogelname mit variierendem Lautwesen verschiedene Eulen-Arten im Skoltlappischen (Paatsjoki, Nuortajärvi) und bei Imandra (Itkonen 1958, 39; Toivonen et al. 1955-81, 95) bezeichnet. Die lappische Vogelbezeichnung geht auf das finnische hyypiä, -piö, -ppiä, hypiä (Berguhu) zurück, und das Wort ist wahrscheinlich eine frühe Entlehung aus dem Ostseefinnischen (Toivonen et al. 1955-81, 95). Der Vogelname hat seine Entsprechung im Karelischen, mit einer ausgeprägten nordsüdlichen Verbreitung (siehe Karte). Mit dem Lautwesen hü̈pie (im Norden) und hübie (im Süden) tritt es so weit nördlich wie Petsamo auf. Im übrigen kommt es im eigentlich karelischen Gebiet in den Gemeinden Kiestinki, Vuokkiniemi, Jyskyjärvi, Tunkua, Rukajärvi, Paatene, Porajärvi, Suojärvi, Korpiselkä, Salmi, Säämäjärvi und Vitele (SMS, Dialektkartensammlung) vor. Das Karelische Wörterbuch (Karjalan 1968, 373) kennt das Wort von Tver.

Sehr nahe den aufgezählten karelischen Gemeinden gibt es den Wortstamm auf finnischem Gebiete in Ortsnamen (Ḧ̈̈piö-, vorwiegend im Norden, und Hï̈piä-, vorwiegend im Süden). Das setzt einen früheren Gebrauch dieses Vogelnamens in den Dialekten voraus. In der südöstlichsten Ausbreitung der Namen in Finnland kommt jedoch auch hyypiä vereinzelt (siehe Karte) als Appellativ vor.

Bevor ich die westfinnische Verbreitung des Dialektwortes kommentiere, werde ich den übrigen Befund in den ostseefinnischen Sprachen erwähnen. 
Wiedemann (Wiedemann 1973 (1893), 1279) hat den Vogelnamen in der

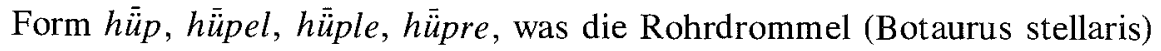
bezeichnet. Jänese-hüp bedeutet dagegen Berguhu (Bubo bubo). Das Institut für Sprache und Literatur in Tallinn (Keele ja Kirjanduse Instituut) kennt das Wort in der ersten Bedeutung und mit den zusätzlichen Formen üüp, üip̀, hüpli mit vereinzeltem Befund in den Gemeinden Jõelähtme, Martna, Urvaste, Kolga-Jaani, Põlva, Räpina, Setumaa, Vasteliina und konzentrierter im Western in Läänemaa und Saaremaa. Im übrigen gibt es den Namen des Berguhus im Olonetzischen (Toivonen et al. 1955-81, 95), Lydischen (Kujola 1944, 82), Wepsischen (Zajceva \& Mullonen 1972, 137), Wotischen (Posti 1980, 81) und den ingrischen Dialekten (Nirvi 1971, 78). Überall hat der Vogel eine hervortretende Rolle als Warnvogel.

Die Variation im Lautwesen im Westen $(h-, j$ - oder der Entfall von initialem Konsonant) kann laut Toivonen et al. auf den onomatopoetischen Charakter des Wortes zurückgeführt werden und bei den ostseefinnischen Sprachen auf Einfluss von huuhkaja (mit Varianten), welches das häufigste Wort für Berguhu ist. Wiklund hat das Bemerkenswerte, dass langes finnisches $-y y$ - in den westlichen lappischen Entsprechungen des Wortes kurzes - $i$ - hat, besprochen (Wiklund 1896, 248; vgl. Korhonen 1981, 82).

Hyypiö hat eine sehr interessante und begrenzte Verteilung in den südwestlichen Dialekten in Finnland. Das Wort ist mit seinen Aussprachevarianten auf das Eigentliche Finnland und die naheliegenden Teile von Nyland und dem südlichen Satakunta konzentriert. Ausserhalb von diesem Gebiet habe ich den Ortsnamenbeleg $H \ddot{u} p i a ̈-$ nur in Jalasjärvi im südlichen Österbotten und den Appellativ hüppiö in Ruovesi im Tavastland wiedergefunden. Die Namensglieder Hüpiö- in Himanka, Rantsila und Paltamo müssen als Belege aus einer Randzone des nordfinnischen HüpiöGebiets angesehen werden. Das hat wiederum zweifellos seine sprachliche Anknüpfung an den nordkarelischen Appellativ hüpie (s.o.) und einem früheren Gebrauch des finnischen Dialektwortes hyypiö. Das letztere wird übrigens von Jagdbeschwörungen bestätigt, die am Ende des achtzehnten und Anfang des neunzehnten Jahrhunderts aufgezeichnet worden sind, in welchen die Herrscherin des Waldes Hyypiö, Hyyperö und Hypere genannt wird (Haavio 1967, 80). Die Gemeinden, in denen die Belege aufgezeichnet wurden, liegen im südöstlichen und nördlichen Savolax (u.a. Juva und Kiuruvesi), nahe den Orten, wo die erwähnten Ortsnamen sich finden. Der Name der weiblichen Jagdgöttlichkeit wird für eine volksetymologische Bildung gehalten (Haavio 1967, 82). Die Tatsache, dass sowohl die Vogelbezeichnung wie auch der Ortsnamenbeleg in den zentralen Teilen Finnlands fehlen, ist ein wichtiges Detail in der Wortverteilung. Offenbar hat das ostlappische Vorkommen des Wortes mit karelischem Einfluss zu tun. 
Schon früher habe ich auf den Zusammenhang zwischen den karelischen Appellativen und den ost- und nordfinnischen Ortsnamen verwiesen. Der Name für Berguhu im südwestlichen Finnland wird sprachgeographisch von dem entsprechenden Namen, der südlich und östlich des Finnischen Meerbusens zu finden ist, erhellt. Der Wortkarte entnehmen wir, dass dieser Name für Berguhu während einer Zeitperiode sich von südöstlichen Gruppen ostseefinnischer Sprachen nach Westen und Norden verbreitet hat. Die früh besiedelten südwestlichen Teile Finnlands, Teile des Finnischen Meerbusens und die Gegenden um den Ladogasee mit dem nahebelegenen Karelien, das ein nördliches Hochland hat, haben in anderen Zusammenhängen alte gemeinsame Kulturelemente aufgewiesen. $\mathrm{Zu}$ den südlichen lappischen Dialekten kann der Vogelname nur aus dem südwestlichen Finnland gekommen sein. Die Waldfinnen, die nach Schweden aus dem zentralen Finnland kamen, hatten wahrscheinlich dieses Wort nicht in ihrem Dialekt. In meiner Dissertation über die Verbreitung finnischer Bootstermini und Ortsnamenelemente im Lappischen und ihre äusserste Verbreitung in einer slawischen Kultursphäre, habe ich gerade auf den Finnischen Meerbusen als einen Verbreitungskorridor verwiesen (Korhonen 1982).

Aber was wäre der Grund für eine Entlehnung des aktuellen Wortes ins Lappische? Es kann die sehr starke Stellung des Berguhus als Warnvogel im Volksglauben sein (Tillhagen 1978, 210f.). Im Volkskundearchiv (Kansanrunousarkisto) in Helsinki finden sich eine Menge Beispiele: Wenn der Berguhu (hyyppi) nahe an ein Dorf kam und im Wald mit einer ,,alten" Stimme rief, starb kurz danach eine alte Frau oder ein Mann in einem der Häuser des Dorfes. Wenn er mit junger Stimme rief, starb ein junger Mensch im Dorf" (Pyhäranta 1929). In den Archiven von ULMA, Uppsala, heisst es von Sorsele in Västerbotten: ,,Der Berguhu ist auch ein 'Feigvogel'." Wenn er anfängt, sich in der Nähe eines Hofes aufzuhalten, wird jemand im Hof sterben (1933). Von Jokkmokk wird erzählt, dass ein Mensch im Dorf sterben wird, wenn der Berguhu (lidno) in der Nähe von der Sita (d.h. dem nomadisierenden Dorf) zu schreien anfängt und näher kommt und dann rufend davonfliegt (H. Grundström). Eine ältere Lappenfrau, die ich in Jokkmokk interviewte, erzählte, dass der Berguhu in der Nähe rief, als ihr Mann krank lag. Sie nahm ein glühendes Holzscheit aus dem Feuer und warf es in die Richtung des Vogels und war sicher, dass sie auf diese Art ihren Mann vom Sterben gerettet hatte.

Der Berguhu hält sich am liebsten in der Einöde auf, versteckt sich während des Tages in den Höhlungen der Felsabhänge und zeigt sich bei seiner Jagd in beeindruckender Grösse mit einem Federkleid, das gelb, schwarz und weiss schillert. Er ist der grösste aller Arten seiner Gattung. 
Er kann bis zu 4 Kilo wiegen. All dies hat dazu beigetragen, ihm eine Sonderstellung in den volkstümlichen Vorstellungen zu geben. Am häufigsten hat man den schreckenserregenden Laut des Vogels mit jähem Tod und Unglück verbunden.

Es ist nicht schwer zu verstehen, dass der Berguhu der Hilfsgeist des Zauberers (Skanke 1945, 101-92) und im weitesten Sinne ein Warnvogel wurde. In lappische Gegenden muss der finnische Name des Vogels mit einer Bevölkerung gekommen sein, die tief verwurzelte Vorstellungen von dessen übernatürlichem Charakter hatte. Dieses geschah offenbar schon vor langer Zeit. Vielleicht ist auch anderer Einfluss auf älteren lappischen völkischen Glauben denselben Weg gekommen? Diese Frage zu erörtern, wird der zukünftigen Forschung zufallen.

\section{Literaturverzeichnis}

ABKÜRZUNGEN

$\begin{array}{ll}\text { fi } & \text { finnisch } \\ \text { saL } & \text { lulelappisch } \\ \text { saN } & \text { nordlappisch }\end{array}$

UNGEDRUCKTE QUELLEN

\section{Helsinki}

Kansanrunousarkisto

SMS Suomen murteiden sanakirjan kokoelmat

Dialektkartensammlung

Oslo

OUB Oslo universitetsbibliotek

1465 e, J. Qvigstad

\section{Umeå}

DAUM Dialekt-, ortnamns- och folkminnesarkivet i Umeå 3398 (Tonband) O. Korhonen

3622 K. B. Wiklund 1900

3793 I. Ruong

3844 A. Calleberg 1925-26

\section{Uppsala}

ULMA Dialekt- och folkminnesarkivet i Uppsala

3032 A. Calleberg 1930

16359 B. Collinder 1943

UUB Uppsala universitetsbibliotek

R 649 J. A. Nensén

\section{Gedruckte Quelien und Literatur}

Bäckman, L. 1975. Sájva. (Stockholm Studies in Comparative Religion 13.) Stockholm. 
Collinder, B. 1938. Lautlehre des waldlappischen Dialekts von Gällivare. (Mémoires de la Société Finno-Ougrienne 74.) Helsinki.

Dahlstedt, T. 1976. Tro och föreställningar kring vitra i öure Norrland. Umeå.

Fellman, J. 1903. Anteckningar under min vistelse $i$ Lappmarken 1. Helsingfors.

Friis, J. A. 1887. Lexicon Lapponicum. Christiania.

Grundström, H. 1946-54. Lulelapsk ordbok-Lulelappisches Wörterbuch. (Skrifter utgivna genom Landsmåls- och Folkminnesarkivet i Uppsala C, 1.) Uppsala.

Grundström, H. \& Väisänen, A. O. 1958. Lappische Lieder 1. (Skrifter utgivna genom Landsmåls- och Folkminnesarkivet i Uppsala C, 2.) Uppsala.

Haavio, M. 1967. Suomalainen mytologia. Helsinki.

Halász, I. 1896. Pite lappmarki szótár és nyelvtan. (Svédlapp nyelv 6.) Budapest.

Hasselbrink, G. 1983. Südlappisches Wörterbuch 2. (Schriften des Instituts für Dialektforschung und Volkskunde in Uppsala C, 4.) Uppsala.

Itkonen, T. I. 1946. Heidnische Religion und späterer Aberglaube bei den finnischen Lappen. (Mémoires de la Société Finno-Ougrienne 87.) Helsinki.

- 1958. Koltan-ja Kuolanlapin sanakirja - Wörterbuch des Kolta- und Kolalappischen. (Lexica Societatis Fenno-Ugricae 15, 1.) Helsinki.

Karjalan kielen sanakirja 1. 1968. (Lexica Societatis Fenno-Ugricae 16, 1.) Helsinki.

Kildeskrifter til den Lappiske Mythologi 1. 1903. [Ed. by] J. Qvigstad. (Det Kgl. Norske Videnskabers Selskabs Skrifter 1903, 1.) Trondhjem.

Kolmodin, T. 1914. Folktro, seder och sägner från Pite lappmark. (Lapparna och deras land 3.) Stockholm.

Korhonen, M. 1981. Johdatus lapin kielen historiaan. (Suomalaisen Kirjallisuuden Seuran toimituksia 370.) Helsinki.

Korhonen, O. 1982. Samisk-finska bättermer och ortnamnselement och deras slaviska bakgrund. (Skrifter utg. av Dialekt-, ortnamns- och folkminnesarkivet i Umeå A, Dialekter 3.)

Kujola, J. 1944. Lyydiläismurteiden sanakirja. (Lexica Societatis Fenno-Ugricae 9.) Helsinki.

Lagercrantz, E. 1939. Lappischer Wortschatz 1-2. (Lexica Societatis FennoUgricae 6.) Helsinki.

Lindahl, E. \& Öhrling, J. 1780. Lexicon Lapponicum. Holmiae.

Nielsen, K. \& Nesheim, A. 1962. Lapp dictionary 5. Supplement. (Instituttet for sammenlignende kulturforskning B, 18, 5.) Oslo.

Nirvi, R. E. 1971. Inkeroismurteiden sanakirja. (Lexica Societatis Fenno-Ugricae 18.) Helsinki.

Pettersson, O. P. 1979. Kristoffer Sjulssons minnen. [Ed. by] L. Bäckman \& R. Kjellström. (Acta Lapponica 20.) Stockholm.

Posti, L. 1980. Vatjan kielen Kukkosin murteen sanakirja. (Lexica Societatis Fenno-Ugricae 19.) Helsinki.

Qvigstad, J. 1893. Nordische Lehnwörter im Lappischen. (Christiania VidenskabsSelskabs Forhandlinger 1893, 1.) Christiania.

- 1943. Marginalier. Nordnorske samlinger 5, 2.) Oslo.

Ravila, P. 1934. Reste lappischen Volksglaubens. (Mémoires de la Société FinnoOugrienne 68.) Helsinki.

Schlachter, W. 1958. Wörterbuch des Waldlappendialekts von Malå und Texte zur Ethnographie. (Lexica Societatis Fenno-Ugricae 14.) Stockholm.

Skanke, H. 1945. Epitomes historiae missionis Lapponicae 1. [Ed. by] O. Solberg. Nordnorske samlinger 5, 2. Oslo. 
Tillhagen, C.-H. 1978. Fåglarna i folktron. Stockholm.

Toivonen, Y. H. et al. 1955-81. Suomen kielen etymologinen sanakirja 1-7. (Lexica Societatis Fenno-Ugricae 12, 1-7.) Helsinki.

Wiedemann, F. J. 1973 (1893). Eesti-saksa sõnaraamat - Estnisch-deutsches Wörterbuch. Tallinn.

Wiklund, K. B. 1896. Entwurf einer urlappischen Lautlehre. (Mémoires de la Société Finno-Ougrienne 11, 1.) Helsingfors.

- 1916. Saivo. (Le monde oriental 10.) Uppsala.

Zajceva, M. I. \& Mullonen, M. I. 1972. Slovar' vepsskogo jazyka. Leningrad. 